\title{
Article \\ Transcribed Ultraconserved Regions Are Associated with Clinicopathological Features in Breast Cancer
}

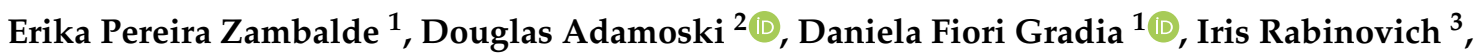 \\ Ana Carolina Rodrigues $\left.{ }^{1}{ }^{(}\right)$, Cristina Ivan ${ }^{4}{ }^{(0)}$, Enilze M. S. F. Ribeiro ${ }^{1}$, George Adrian Calin ${ }^{4,5}$ \\ and Jaqueline Carvalho de Oliveira ${ }^{1, * \mathbb{B}}$
}

\section{check for}

Citation: Zambalde, E.P.; Adamoski,

D.; Gradia, D.F.; Rabinovich, I.;

Rodrigues, A.C.; Ivan, C.; Ribeiro,

E.M.S.F.; Calin, G.A.; Carvalho de

Oliveira, J. Transcribed

Ultraconserved Regions Are

Associated with Clinicopathological

Features in Breast Cancer.

Biomolecules 2022, 12, 214. https://

doi.org/10.3390/biom12020214

Academic Editor: Prakash Kulkarni

Received: 11 December 2021

Accepted: 22 January 2022

Published: 26 January 2022

Publisher's Note: MDPI stays neutral with regard to jurisdictional claims in published maps and institutional affiliations.

Copyright: (c) 2022 by the authors Licensee MDPI, Basel, Switzerland. This article is an open access article distributed under the terms and conditions of the Creative Commons Attribution (CC BY) license (https:// creativecommons.org/licenses/by/ $4.0 /)$.
1 Laboratory of Human Cytogenetics and Oncogenetics, Department of Genetics, Universidade Federal do Paraná, Curitiba 81531-980, PR, Brazil; erikazambaldi@gmail.com (E.P.Z.); danielagradia@ufpr.br (D.F.G.); ana.acr.rodrigues@gmail.com (A.C.R.); enilzeribeiro@gmail.com (E.M.S.F.R.)

2 Brazilian Biosciences National Laboratory (LNBio), Brazilian Center for Research in Energy and Materials (CNPEM), Campinas 13083-970, SP, Brazil; douglas.adamoski@gmail.com

3 Hospital Nossa Senhora das Graças, Centro de Doenças da Mama, Curitiba 80810-040, PR, Brazil; iris_rbv@yahoo.com.br

4 Translational Molecular Pathology Department, The University of Texas MD Anderson Cancer Center Houston, TX 77230, USA; CIvan@mdanderson.org (C.I.); gcalin@mdanderson.org (G.A.C.)

5 Center for RNA Interference and Non-Coding RNAs, The University of Texas MD Anderson Cancer Center, Houston, TX 77230, USA

* Correspondence: jaqueline.carvalho@ufpr.br

\begin{abstract}
Ultraconserved regions (UCRs) are 481 genome segments, with length longer than $200 \mathrm{bp}$, that are $100 \%$ conserved among humans, mice, and rats. The majority of UCRs are transcriptionally active (T-UCRs) as many of them produce non-coding RNAs. In a previous study, we evaluated the expression level of T-UCRs in breast cancer (BC) patients and found that $63 \%$ of transcripts correlated with some clinical and/or molecular parameter of BC. In this study, we delved into the expression levels of 12 T-UCRs and correlated them with clinicopathological parameters, immunohistochemical markers, and overall survival in two breast cancer cohorts: TCGA and Brazilian patients. We found that uc.268 is more expressed in TCGA patients under 40 years of age, associated with progesterone receptor (PR) and estrogen receptor (ER), and its high expression is found in luminal A. Lower uc.84 and uc. 376 were respectively observed in metastatic and stage IV tumors associated with good prognostic in luminal B. Moreover, uc. 84 was only related to the HER2+, while uc.376 was related to ER+ and PR+, and HER2+. A panel composed of uc.147, uc.271, and uc.427 distinguished luminal A from triple negative patients with an AUC of 0.9531 (sensitivity $92.19 \%$ and specificity $86.76 \%$ ). These results highlight the potential role of T-UCRs in BC and provide insights into the potential application of T-UCRs as biomarkers.
\end{abstract}

Keywords: T-UCRs; breast cancer (BC); lncRNAs

\section{Introduction}

Human genome sequencing demonstrated that only $2 \%$ of DNA are protein-coding genes. The remaining 98\% constitutes what was previously called the "dark matter" of molecular biology and includes hidden information mainly responsible for phenotype regulation. Dark matter has become a new field to explore [1]. Nowadays, it is proven that this region can be transcribed into distinct RNAs that have an impact on gene regulation [2].

Those RNAs were named non-coding RNAs (ncRNAs), which include a variety of molecules and are subdivided into two major groups: miRNAs (with a sequence less than $200 \mathrm{bp}$ ) and long non-coding RNA (with a sequence of more than $200 \mathrm{bp}$ ) [3].

Ultraconserved regions (UCRs) are regions of conserved DNA segments, which have $100 \%$ identity among humans, rats, and mice [4]. Most of these regions are transcriptionally 
active (T-UCRs), and $80 \%$ are transcribed from genomic elements located in both intraand intergenic regions, representing a class of long-non-coding RNAs [5]. The extreme conservation of T-UCRs in non-coding regions and the significant number of deregulated T-UCRs associated with human diseases may be a sign of their importance in physiologic and pathophysiologic processes [6,7]. Most UCRs are known to be transcribed ultraconserved regions (T-UCRs) in normal and malignant tissues [6]. In addition, T-UCRs are more frequently found in cancer-associated genomic regions (CAGRs), and the differential expression of T-UCRs has been described in several types of cancer [8]. Several studies have identified distinct signatures in human carcinomas, such as hepatocellular (HCC) [9-11], neuroblastoma (NB) [5], chronic lymphocytic leukemia (CLL) [6], prostate cancer (PC) [12], and bladder cancer (BlaC) [13]. T-UCRs expression profiles can be a useful tool to differentiate human cancer types and improve their diagnosis and prognosis. In breast cancer (BC), however, the utility of T-UCRs as a biomarker is still underexplored.

$\mathrm{BC}$ is the most commonly diagnosed type of cancer globally and the leading cause of cancer death among women worldwide [14]. In addition, BC is a heterogeneous disease with a range of clinical and molecular characteristics [15]. Based on immunohistochemical analysis, BC is classified into four main groups: luminal A, luminal B, HER2 (Human Epidermal Growth Factor Receptor 2) enriched, and triple-negative [16,17]. This classification is essential for prognosis and treatment decisions. The triple-negative is the most challenging group to treat, but new targeted therapies are becoming available, e.g., PARP inhibitors in BRCA mutated patients [18]. Monoclonal antibody therapy has excellent results in HER2 enriched patients [19]. Luminal A and luminal B patients can benefit from hormone therapies. Moreover, $\mathrm{BC}$ can also be categorized according the expression profile of 50 genes using partition around medoids, a method called PAM50. In this classification BC is stratified into five groups: luminal A, luminal B, HER2 enriched, basal, and normal-like $[20,21]$.

Despite the $\mathrm{BC}$ general classification system and the existing guidelines for the stratification of patients inside each group, the wide range of diversity observed in each subtype demands additional molecular markers to offer personalized therapy and improve BC survival rates [22,23]. In addition, only four T-UCRs have been strongly associated with BC: uc.38, downregulated BC patients [24], uc.63, upregulated in luminal A patients associated with poor prognosis [25], lnc-uc.147, associated with poor prognosis in luminal A [26], and uc.51, associated to metastasis [27].

In a previous study from our group, we evaluated the expression level of all 481 T-UCRs in TCGA (The Cancer Genome Atlas) BC patients and found that $63 \%$ of transcripts correlated with some clinical and/or molecular parameter of BC [26]. Including a T-UCR transcriptional portrait, 12 T-UCRs (uc.84, uc.138, uc.147, uc.193, uc.268, uc.271, uc.311, uc.376, uc.378, uc.427, uc.456, uc.475) were suggested to have great potential as biomarkers in BC, but these molecules were not further investigated [26]. The present study aimed to evaluate the 12 T-UCRs in the TCGA data and the Brazilian cohort. We also associated the T-UCRs with several parameters such as metastasis, age, overall survival, and positivity of receptors. All the evidence found suggests that T-UCRs should be explored as a prognostic marker and target for therapies in BC.

\section{Materials and Methods}

\subsection{Breast Cancer Patients}

This study was approved by the Ethical Committee in Research of the Federal University of Paraná (UFPR) (CONEP: 19870319.3.0000.0102), and all individuals signed a written informed consent form. Tumor samples from 102 breast cancer patients (30 luminal A, 30 luminal B, 9 HER2-enriched, and 33 triple-negative) were collected at Hospital Nossa Senhora da Graças, Curitiba, Paraná, Brazil. Tissue samples were obtained from fresh surgical specimens later frozen in RNA later and stored at $-80^{\circ} \mathrm{C}$. The subtypes of all samples were immunohistochemically confirmed. Patients' clinicopathological characteristics are listed in Table 1 and Supplementary Table S1. 
Table 1. Clinicopathological parameters of Brazilian breast cancer patients.

\begin{tabular}{|c|c|c|c|}
\hline Parameters & Categorization & Sample Size & $\%$ \\
\hline \multirow[t]{4}{*}{ Age at diagnosis } & $\begin{array}{l}\text { Median } 53 \text { years } \\
\text { (range: } 25-90 \text { ) }\end{array}$ & & \\
\hline & $\leq 53$ & 51 & 50.0 \\
\hline & $>53$ & 48 & 47.0 \\
\hline & Unknown & 3 & 2.9 \\
\hline \multirow[t]{3}{*}{ Estrogen receptor status } & Positive & 59 & 57.8 \\
\hline & Negative & 43 & 42.2 \\
\hline & Unknown & - & - \\
\hline \multirow[t]{3}{*}{ Progesterone receptor status } & Positive & 59 & 57.8 \\
\hline & Negative & 43 & 42.2 \\
\hline & Unknown & - & - \\
\hline \multirow[t]{3}{*}{ HER2 status } & Positive & 55 & 53.9 \\
\hline & Negative & 45 & 44.1 \\
\hline & Unknown & 2 & 1.9 \\
\hline \multirow[t]{3}{*}{ Proliferation index (Ki-67) } & $\leq 15 \%$ & 33 & 32.4 \\
\hline & $>15 \%$ & 67 & 65.7 \\
\hline & Unknown & 2 & 1.9 \\
\hline \multirow[t]{5}{*}{ Immunohistochemical Subtype } & Luminal A & 30 & 29.4 \\
\hline & Luminal B & 30 & 29.4 \\
\hline & HER2+ & 9 & 8.8 \\
\hline & Triple Negative & 33 & 32.4 \\
\hline & Unknown & - & - \\
\hline \multirow[t]{3}{*}{ Lymph node status } & Positive & 30 & 29.4 \\
\hline & Negative & 57 & 55.9 \\
\hline & Unknown & 15 & 14.7 \\
\hline Distance Metastasis & & 11 & 10.7 \\
\hline Death & & 3 & 2.9 \\
\hline
\end{tabular}

HER2: human epidermal growth factor receptor 2.

\subsection{RNA Extraction, $c D N A$ Synthesis, and RT-qPCR}

RNA from tissues was extracted using the Quick RNA Miniprep Kit (Zymo Research, Irvine, CA, USA). All RNAs were treated with DNase (Ambion, Austin, TX, USA). RNA quality and concentration were assessed using the nanodrop ND-1000 instrument (NanoDrop Technologies, Thermo Scientific, Wilmington, DE, USA) and Bioanalyzer (Agilent, Santa Clara, CA, USA). cDNA was synthesized using the SuperScript III cDNA kit (Invitrogen, Waltham, MA, USA), and diluted cDNA was used for RT-PCR analysis using iQ SYBR Green Supermix (Bio-Rad, Richmond, CA, USA) with the appropriate primers (Supplementary Table S2). The MCF-7 cell line was used as a calibrator sample. The $2^{-\Delta \Delta \mathrm{Ct}}$ method was used to calculate the relative abundance of RNA genes compared with two of the following genes: the TBP and $\mathrm{u} 6$ expression. All experiments were performed with a RT-control to identify DNA contamination.

\subsection{TCGA Data}

The statistical analyses for the TCGA data were performed in R software (version 3.4.1, R, Vienna, Austria) (http:///www.r-project.org/ (accessed on 14 January 2022)), and the statistical significance was defined as a $p$-value less than 0.05 . The ultraconserved elements' (UCR) sequences in the human genome, their genomic coordinates in GRCh34/hg16, as well their type (exonic, nonexonic) and the closest gene were retrieved from https: / / users.soe.ucsc.edu/ jill/ultra.html (accessed on 17 November 2021) [4]. We used UCSC Genome Browser LiftOver to lift the coordinates from GRCh34/hg16 to GRCh37/hg19 assembly. The expression of the ultraconserved regions, quantified as RPKM (reads per kilobase of transcript per million mapped reads) in primary tumors, were retrieved from TANRIC (https://ibl.mdanderson.org/tanric/_design/basic/main.html (accessed on 10 January 2022)) [28]. We downloaded clinical information for the TCGA patients 
with invasive breast carcinoma (BRCA) from the cbioPortal (http: / / www.cbioportal.org / (accessed on 14 January 2022)), (cohort: TCGA provisional). Hormonal marker status for Estrogen Receptor (ER), Progesterone Receptor (PR), and Human Epidermal growth factor Receptor 2 (HER2) were defined from TCGA's IHC data. The PAM50 subtype of the samples was retrieve from [29]. Updated TCGA primary end point data as survival status and follow-up time, were obtained from [30]. For overall survival (OS): overall survival event included death from any cause. For progression-free interval (PFI), event considered patients having new tumor event whether it was a progression of disease, local recurrence, distant metastasis, new primary tumors all sites, or died with the cancer with or without new tumor event.

We obtained 827 cases with UC expression and clinical information. From the Genomic Data Commons Data Portal, we downloaded FPKM (fragments per kilobase of transcript per million mapped reads) files to retrieve gene expression quantification data for the host genes of the ultraconserved regions in the same tumors. The log2-transformation was applied to the mRNA data.

\subsection{Statistical Analysis}

The association between the variables analyzed and T-UCRs expression levels was determined by non-parametric test (Mann-Whitney preceded by Kruskal-Wallis test when multiple comparisons were performed). The relationship between overall survival and covariates (mRNA expression levels and clinical parameters, such as age and stage) was examined through a Cox proportional hazard model. A multivariate Cox proportional hazard model was fitted, including the clinical parameters and mRNA expression significance in the univariate analysis. These analyses were conducted for the breast cancer cohorts and separately on each subtype as defined by PAM50 or immunohistochemistry. The adjusted $p$-value of $<0.05$ was considered significant. Receiver operating characteristic (ROC) curves were calculated based on RQ values. For combined ROC curves, a binary logistic regression was calculated using IBM SPSS Statistics (IBM SPSS Statistics Inc., Armonk, NY, USA). We use the software IBM SPSS Statistics to perform the binary logistic. In this analysis, we used the expression data already obtained. We selected as dependent variable the tumor samples (if we are analyzing tumor versus non-tumor) or luminal A samples (if we are analyzing luminal A versus TNBC). Subsequently, we selected the expression levels of the T-UCRs as the variable that we wish to combine into the covariates deriving Cox regression $\beta$ values for each T-UCR, which were used as weighting factors to derive an overall score of combined T-UCRs expression for each patient. This score were used as the entry for ROC analysis. The true positive rate (sensitivity) versus the false positive rate (1-specificity) were plotted at various threshold settings, and the optimal cutoff threshold was calculated using Youden's index (highest sensibility plus specificity).

\section{Results}

\subsection{Differential Expression of T-UCRs in BC Samples}

All T-UCRs analyzed had expression detected in more than $80 \%$ of the samples analyzed in both cohorts. Analysis of the differential expression of T-UCRs uc.84, uc.138, uc. 147 , uc. 193 , uc. 268 , uc. 271 , uc.311, uc.376, uc.378, uc.427, uc. 456 , and uc. 475 in TCGA BC tumor samples $(n=827)$ versus non-tumor $(n=105)$ demonstrated that 10 T-UCRs (uc.138, uc.147, uc.193, uc.268, uc.271, uc.311, uc.376, uc.378, uc.427, uc.456) had differential expression (Figure 1). The T-UCRs uc.138, uc.311, uc.376, and uc.456 were down expressed while uc.147, uc.193, uc.268, uc.271, uc.378, and uc.427 were highly expressed in the tumor samples.

The diagnostic potential of T-UCRs was analysed through receiver operating characteristic (ROC) curves (Figure 2). The T-UCRs uc.147 and uc.271 had the highest area under the curve (AUC) of 0.64 (uc.147: sensitivity of $61.32 \%$ and specificity of $63.81 \%$; uc. 271 : sensitivity of $60.67 \%$ and specificity of $68.60 \%$ ), followed by uc. 268 and uc. 427 , both with an AUC of 0.60 (uc.268: sensitivity of $56.47 \%$ and specificity of $68.57 \%$ and uc.475: sensitivity 
of $49.74 \%$ and specificity of $74.47 \%$ ) (Figure 2). By combining these four T-UCRs, AUC increase to 0.74 , with $65.42 \%$ sensitivity and $73.33 \%$ specificity (Figure $2 \mathrm{E}$ ). Interestingly, when we create a panel combining all 12 T-UCRs, AUC rises to 0.93 along with higher sensitivity and specificity $(85.37 \%$ and $85.71 \%$, respectively), indicating a great association of these T-UCRs and breast cancer (Figure 2F). Another combination of seven T-UCRs (uc.138, uc.147, uc.268, uc.271, uc.311, uc.427, and uc.475) also demonstrated high AUC (0.92) and good sensitivity (83.09\%) and specificity (87.62\%), which indicates that a panel of these seven T-UCRs could be enough for the BC diagnostic (Figure 2G). Other combinations were also tested, but those aforementioned represent the best combinations of the T-UCRs associated with BC.
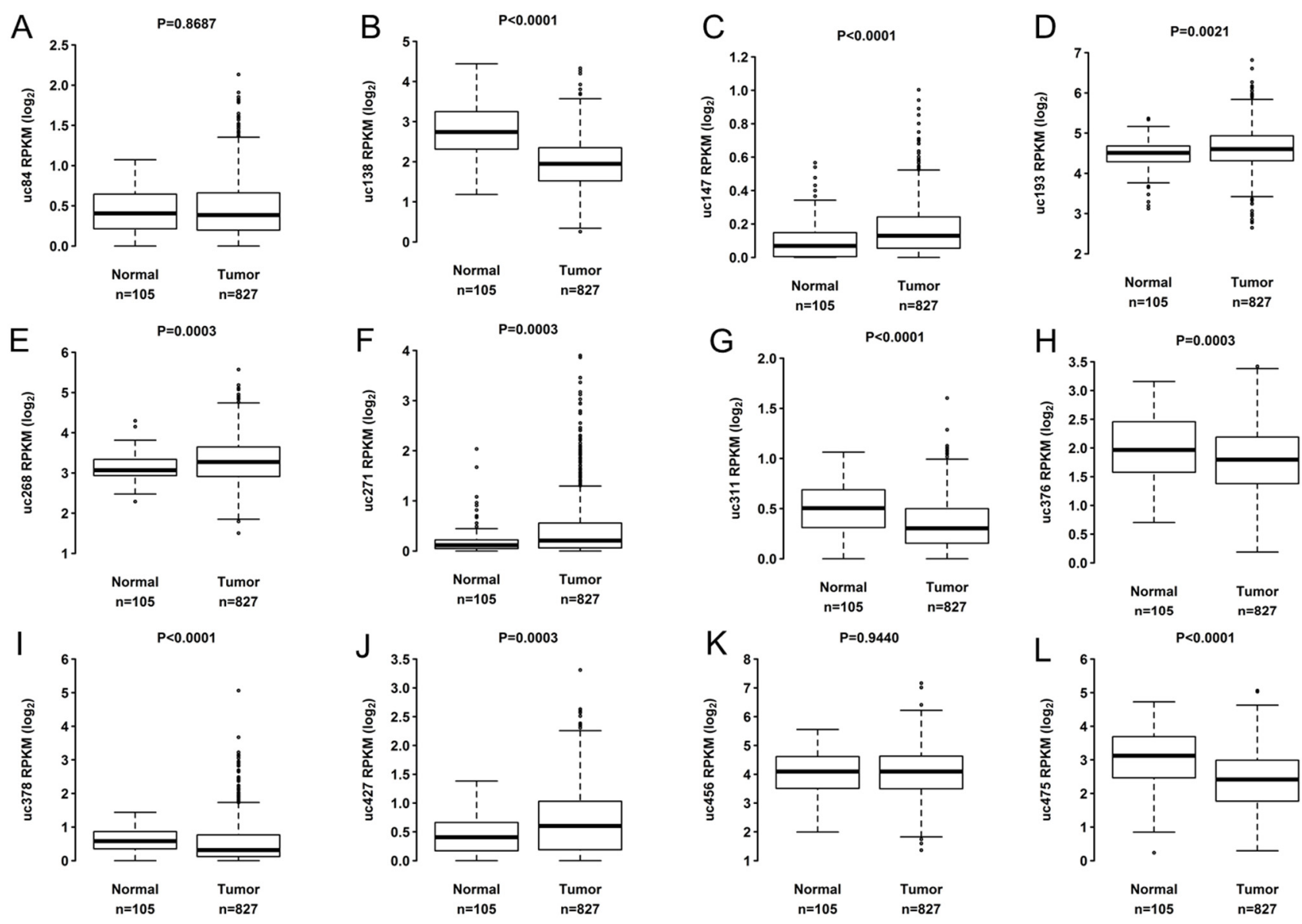

Figure 1. Expression levels of 12 T-UCRs. Comparison of expression levels of 12 T-UCRs in tumorous versus non- tumorous BC samples from TCGA. Differential expression level of (A) uc.84, (B) uc.138, (C) uc.147, (D) uc.193, (E) uc.268, (F) uc.271, (G) uc.311, (H) uc.376, (I) uc.378, (J) uc.427, (K) uc.456 and $(\mathrm{L})$ uc.475 in tumorous versus non-tumorous. Each dot represents outlier patient.

\subsection{Differential Expression of T-UCRs in BC Subtypes}

Classifying subtypes in BC is essential for prognosis and treatment decisions. All $12 \mathrm{~T}$ UCRs from TCGA cohort (Figure 3) and two T-UCRs (uc.147 and uc.193) in the Brazilian (102) cohort presented differential expression among subtypes.

We also tested the potential of T-UCRs to discriminate the subtypes luminal A (better prognostic) and triple-negative (worse prognostic). We noted that uc.147 has better potential do differentiate both subtypes in both cohorts, TCGA and Brazilian, with an AUC of 0.822 (sensitivity of $85.94 \%$ and specificity of $66.76 \%$ ) and 0.815 (sensitivity of $88.89 \%$ and specificity of $66.67 \%$ ) respectively (Figure $4 \mathrm{~A}, \mathrm{~B}$ ). For the TCGA cohort, two more T-UCRs were good markers: uc.271 (AUC: 0.865 ; sensitivity of $85.16 \%$ and specificity of $78.85 \%$ ) and uc.427 (AUC: 0.875 ; sensitivity of $85.16 \%$ and specificity of 78.85 ) (Figure 4C,D). 

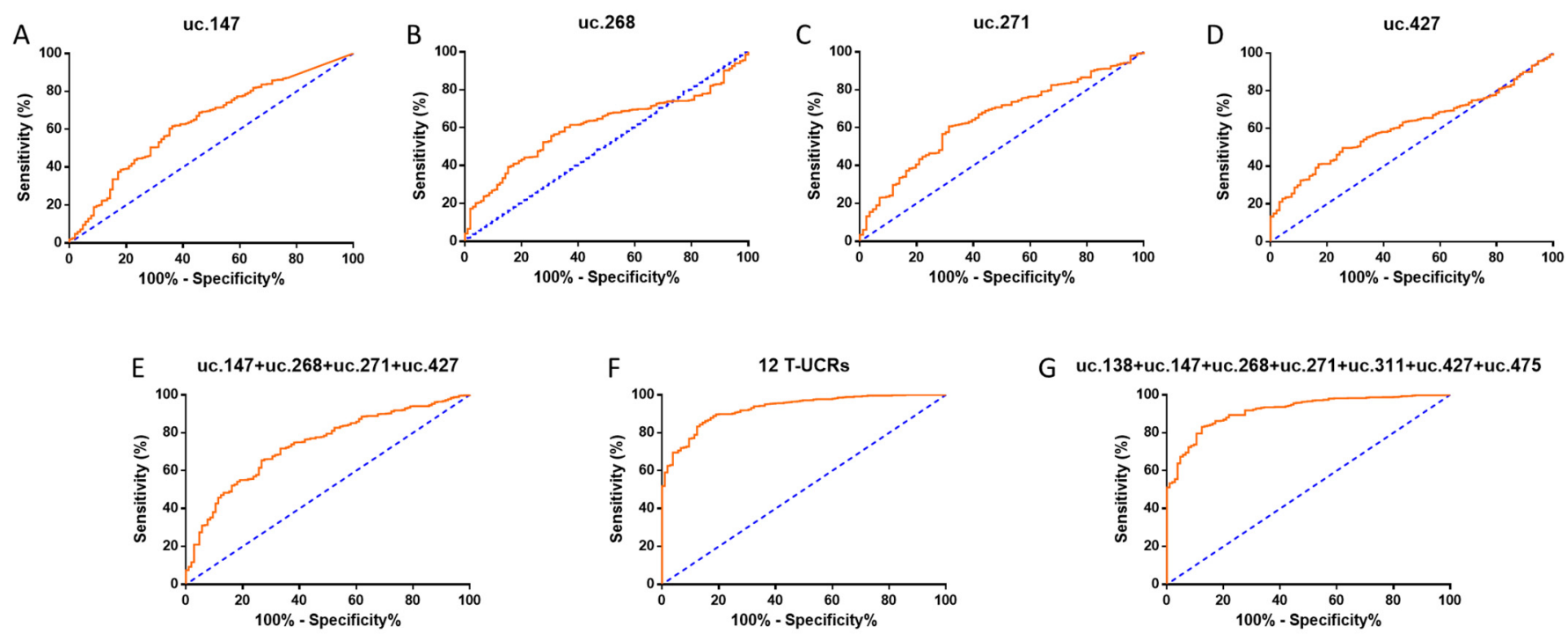

Figure 2. Individual and combined receiver operating characteristic (ROC) curves from T-UCRs, indicating diagnostic potential panels. (A-D) BC prognostics accuracy calculated for uc.147 TCGA (A), uc.268 TCGA (B), uc.271 TCGA (C), and uc.427 TCGA (D). (E-G) Combined ROC curves of uc. 147 + uc. 268 + uc. 271 + uc. 427 (E), all 12 T-UCRs (F), and uc. 138 + uc. 147 + uc. $268+$ uc. $271+311+$ uc. $427+$ uc.475 (G). ROC curves calculated based on relative quantification (RQ) values. All $p$ values are under 0.05
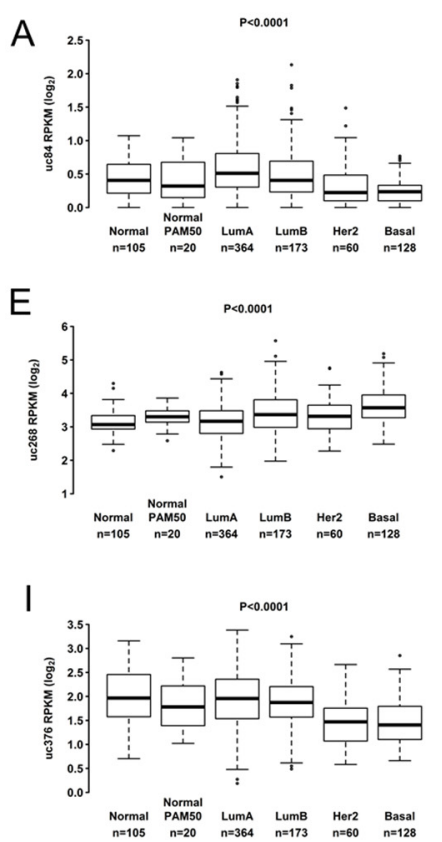

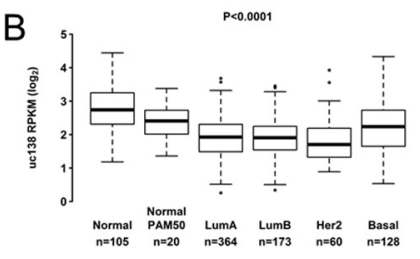

F
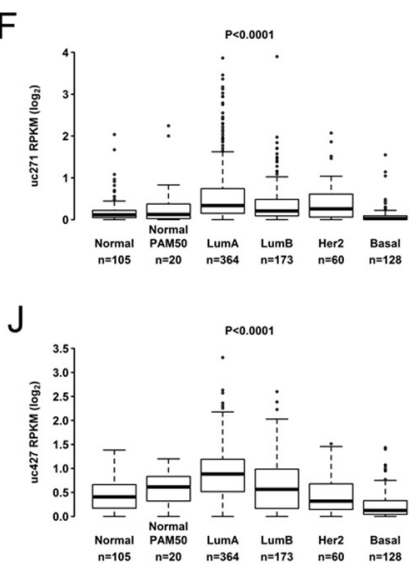
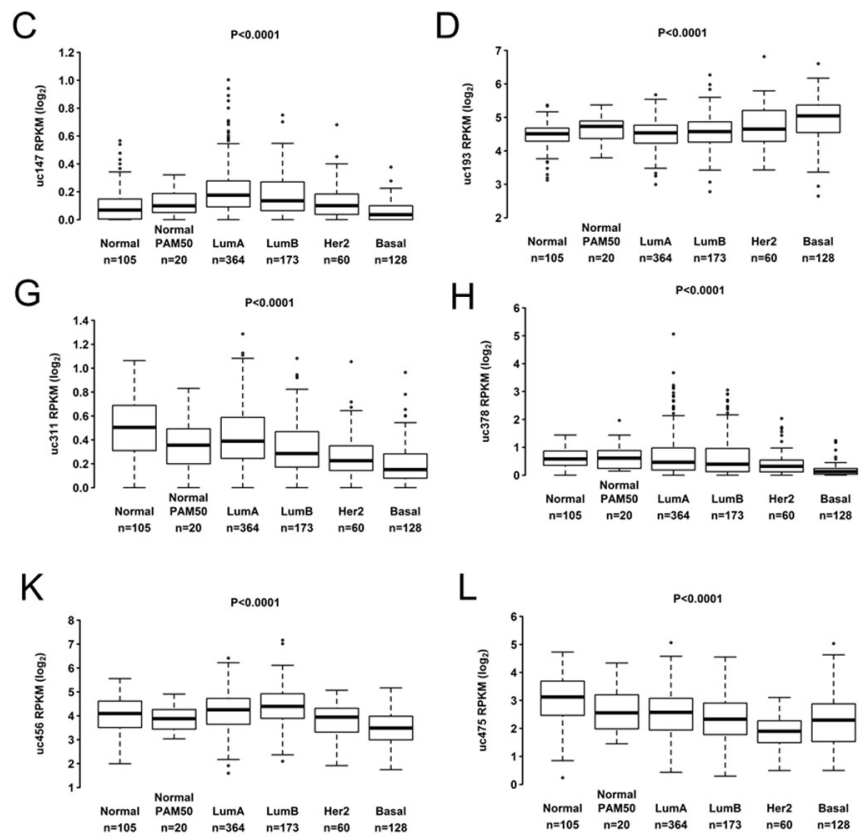

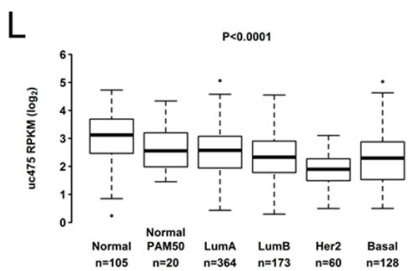

Figure 3. Expression levels of 12 T-UCRs in all breast cancer subtypes from TCGA patients: Normal, Normal PAM50, Luminal A (LumA), Luminal B (LumB), HER2 overexpression (HER2+), and Basal. (A) Differential expression level of uc.84, (B) uc.138, (C) uc.147, (D) uc.193, (E) uc.268, (F) uc.271, (G) uc.311, (H) uc.378, (I) uc.376, (J) uc.427, (K) uc.456 and (L) uc.475 according to PAM50 BC subtypes.

Interestingly, the discriminatory accuracy of the ROC curve is improves combined with the expression levels of uc.147, uc.271, and uc.427, which also presented good AUC values. The combined AUC of these T-UCRs improved to 0.9531 (Figure 4E), as well as the sensitivity and specificity values $(92.19 \%$ and $86.76 \%$, respectively). Together, these results indicate that the selected T-UCRs, either individually or combined, can distinguish 
$\mathrm{BC}$ between the luminal A and TNBC (triple negative breast cancer) subtypes with high sensitivity and specificity.
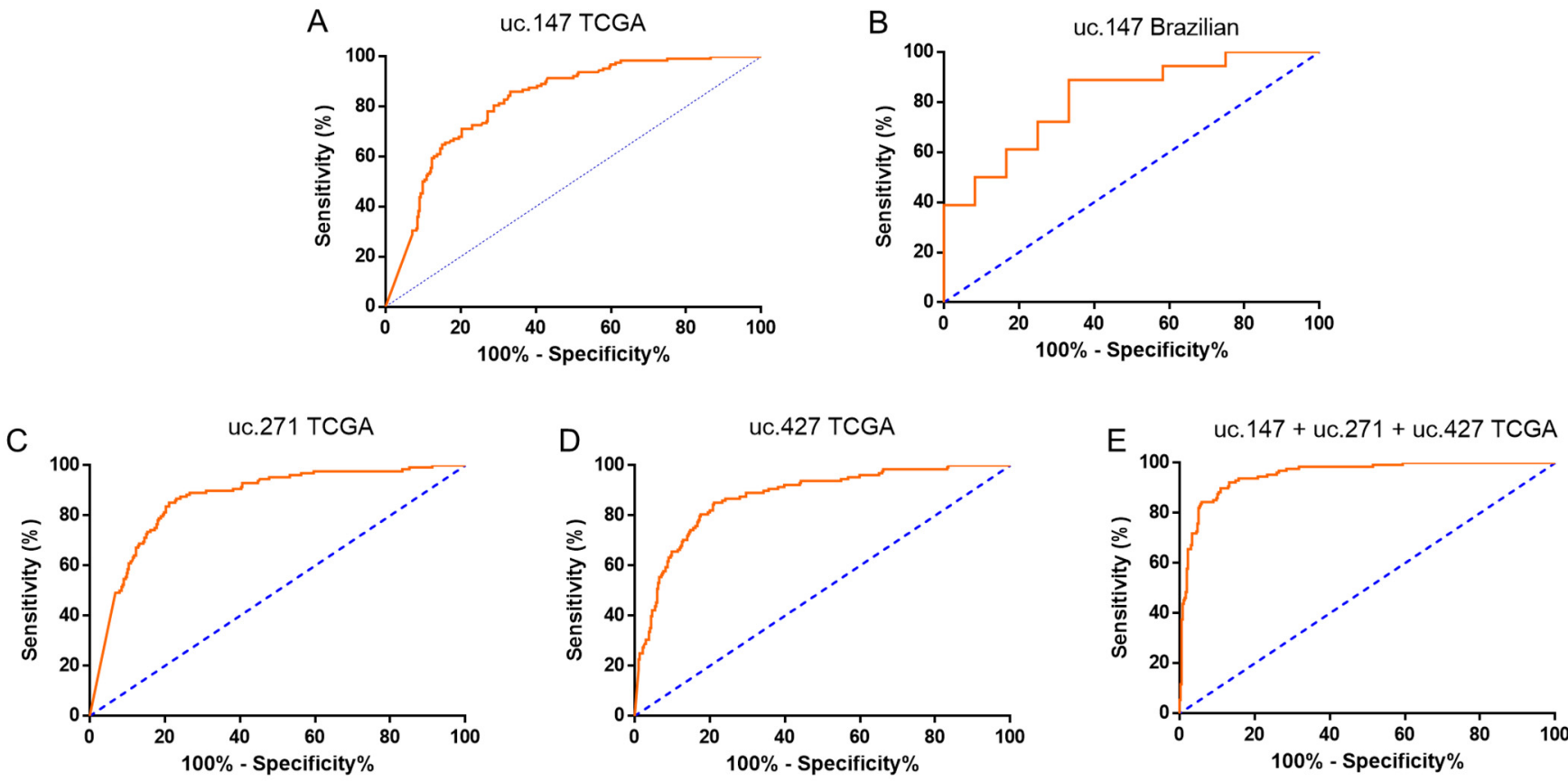

Figure 4. Individual and combined receiver operating characteristic (ROC) curves fromT-UCRs, indicating prognostic potential panels. BC prognostics accuracy calculated for uc.147 TCGA (A), uc.147 Brazilian cohort (B), uc.271 TCGA (C), and uc.427 TCGA (D). (E) BC prognostics accuracy calculated for uc.147 from Brazilian cohort. ROC curves calculated based on relative quantification (RQ) values.

\subsection{Association of T-UCRs Expression with Clinicopathological Parameters of BC}

Immunohistochemical analysis was used to stratify BC and therapy decisions. Most T-UCRs analyzed in TCGA have differential expression according to progesterone and estrogen receptors. Out of the 12 T-UCRs analyzed, 10 (uc.147, uc.193, uc.268, uc.271, uc.311, uc.376, uc.378, uc.427, uc.456, uc.475) showed association with the progesterone receptor (Supplementary Figure S1), 11 (uc.138, uc.147, uc.193, uc.268, uc.271, uc.311, uc.376, uc.378, uc.427, uc.456, uc.475) with the estrogen receptor (Supplementary Figure S2), and four (uc.84, uc.138, uc.376 and uc.475) with the HER2 status (Supplementary Figure S3).

In TCGA data, uc. 268 is more expressed in diagnosed patients who are under 40 years old (Figure 5A). A lower expression of uc.84 was observed in patients with metastasis (Figure 5B). In addition, stage IV tumors showed a lower expression of uc.376 (Figure 5C). These results suggest that uc. 268 could be helpful to identify risk for earlier BC, while uc. 84 and uc.376 could indicate less aggressive cancer. In the Brazilian cohort, no association was found, possibly due to a small number of samples.

\subsection{T-UCRs Expression in BC Correlates with Patients' Overall Survival}

Using univariate analysis, we noted that uc.193 is associated with overall survival (OS) in breast cancer. Using a multivariate analysis, we demonstrated that high uc.193 expression is an independent prognostic marker of poor survival for BC (HR $=1.75$, IC 95\%: $1.21-2.52, p<0.01)$ (Table 2). We also analyzed different subtypes and for the hormonal and HER2 receptors. 
A

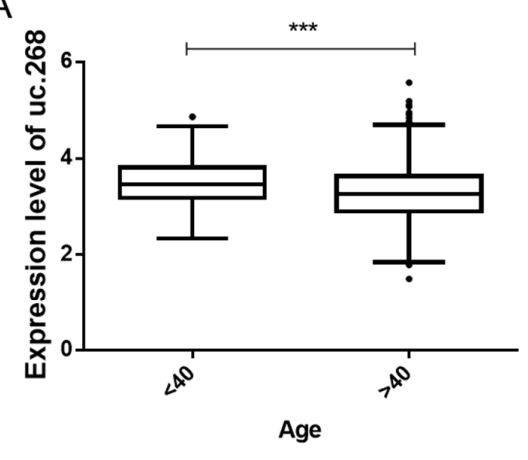

C

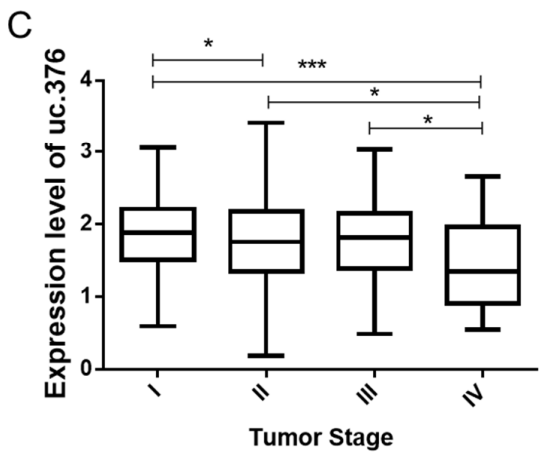

B

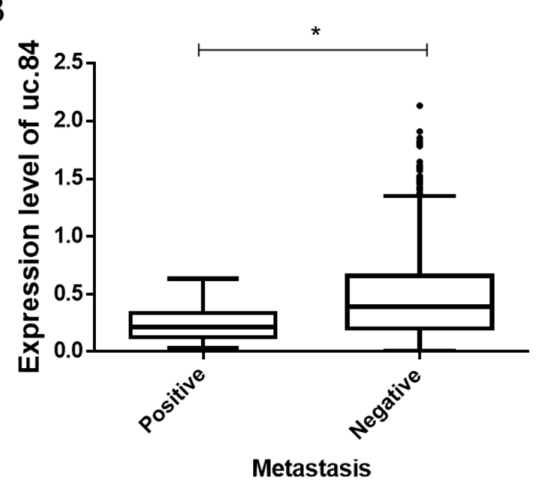

Figure 5. Comparison of T-UCRs expression to clinicopathological data from TCGA data. (A) Differential expression level of uc.268 between the patients' ages $(p<0.001)$. (B) Significant down-relative expression of uc.84 patients with metastasis compared to patients negative for metastasis $(p<0.05)$. (C) Significant reduction in uc.376 $(p<0.05)$ between tumor stage I, II, III, and IV. ${ }^{*} p<0.05$, and *** $p<0.001$.

Table 2. Summary of univariate and multivariate Cox regression analysis of al T-UCRs in overall patients survival.

\begin{tabular}{|c|c|c|c|c|c|c|c|c|c|}
\hline & \multicolumn{5}{|c|}{ Univariate Analysis } & \multicolumn{4}{|c|}{ Multivariate Analysis } \\
\hline & Variable & HR & Lower 0.95 & Upper 0.95 & $p$-Value & HR & Lower 0.95 & Upper 0.95 & $p$-Value \\
\hline \multirow{14}{*}{ 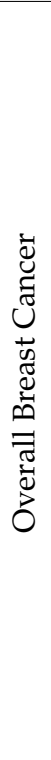 } & Age & 1.03 & 1.02 & 1.05 & $1.92 \times 10^{-5 *}$ & 2.34 & 1.55 & 3.52 & $5.30 \times 10^{-5 *}$ \\
\hline & Stage & 2.34 & 1.55 & 3.52 & $5.30 \times 10^{-5 *}$ & 1.04 & 1.02 & 1.05 & $5.9 \times 10^{-7 *}$ \\
\hline & uc. 84 & 1.19 & 0.74 & 1.92 & 0.46 & & & & \\
\hline & uc. 138 & 1.11 & 0.84 & 1.46 & 0.48 & & & & \\
\hline & uc.147 & 2.49 & 0.79 & 7.83 & 0.12 & 1.75 & 1.21 & 2.52 & $0.0026^{*}$ \\
\hline & uc.193 & 1.44 & 1.04 & 1.98 & 0.03 * & & & & \\
\hline & uc. 268 & 1.19 & 0.86 & 1.64 & 0.29 & & & & \\
\hline & uc. 271 & 0.87 & 0.64 & 1.18 & 0.36 & & & & \\
\hline & uc.311 & 0.64 & 0.31 & 1.34 & 0.23 & & & & \\
\hline & uc.376 & 1.09 & 0.79 & 1.49 & 0.61 & & & & \\
\hline & uc.378 & 1.03 & 0.77 & 1.39 & 0.83 & & & & \\
\hline & uc. 427 & 0.99 & 0.71 & 1.38 & 0.95 & & & & \\
\hline & uc. 456 & 1.18 & 0.95 & 1.48 & 0.14 & & & & \\
\hline & uc. 475 & 1.19 & 0.95 & 1.48 & 0.12 & & & & \\
\hline
\end{tabular}


In luminal A, a subtype usually associated with better prognosis, the expression of six T-UCRs (uc.84, uc.147, uc.193, uc.268, uc.456 and uc.475) was associated with survival time in uni- and multivariate analysis, highlighting uc.193 with a 6.54 HR (Supplementary Table S3).

Another five T-UCRs (uc.138, uc.147, uc.311, uc.376, and uc.456) were indicated as luminal B subtype correlated factors, an intermediate subtype in BC. In the HER2 subtype, which is an aggressive subtype but has targeted therapy available, the uc.193 was shown to associated to this subtype (Table 3). No T-UCRs analyzed were associated with overall survival in triple-negative.

These results demonstrated a relation between the T-UCRs and the survival rate in different subtypes, evidencing that the T-UCRs could also be used as a prognostic marker.

Table 3. Summary of univariate Cox regression analysis of T-UCRs in overall survival among subtypes.

\begin{tabular}{|c|c|c|c|c|c|}
\hline & \multirow[b]{2}{*}{ Variable } & \multicolumn{4}{|c|}{ Univariate Analysis } \\
\hline & & HR & Lower 0.95 & Upper 0.95 & $p$-Value \\
\hline \multirow{14}{*}{ 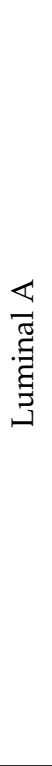 } & Age & 1.032 & 1.011 & 1.054 & $0.003 *$ \\
\hline & Stage & 1.726 & 0.916 & 3.254 & 0.091 \\
\hline & uc. 84 & 2.202 & 1.170 & 4.145 & 0.014 * \\
\hline & uc.138 & 1.555 & 0.980 & 2.466 & 0.061 \\
\hline & uc.147 & 8.042 & 1.891 & 34.207 & $0.005 *$ \\
\hline & uc.193 & 2.629 & 1.320 & 5.236 & $0.006^{*}$ \\
\hline & uc. 268 & 1.761 & 1.029 & 3.016 & $0.039 *$ \\
\hline & uc. 271 & 0.764 & 0.499 & 1.168 & 0.214 \\
\hline & uc.311 & 0.949 & 0.330 & 2.727 & 0.922 \\
\hline & uc.376 & 1.454 & 0.870 & 2.429 & 0.153 \\
\hline & uc.378 & 1.217 & 0.840 & 1.763 & 0.298 \\
\hline & uc. 427 & 1.161 & 0.693 & 1.945 & 0.571 \\
\hline & uc. 456 & 1.604 & 1.085 & 2.372 & 0.018 * \\
\hline & uc. 475 & 1.588 & 1.089 & 2.317 & 0.016 * \\
\hline \multirow{14}{*}{ 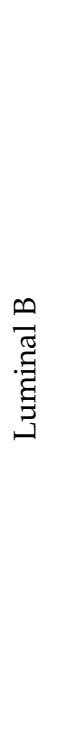 } & Age & 1.024 & 0.994 & 1.055 & 0.112 \\
\hline & Stage & 2.108 & 0.959 & 4.632 & 0.063 \\
\hline & uc. 84 & 0.220 & 0.042 & 1.140 & 0.071 \\
\hline & uc.138 & 0.530 & 0.288 & 0.973 & 0.041 * \\
\hline & uc.147 & 0.002 & 0.000 & 0.080 & 0.001 * \\
\hline & uc.193 & 1.811 & 0.867 & 3.784 & 0.114 \\
\hline & uc. 268 & 0.688 & 0.354 & 1.336 & 0.269 \\
\hline & uc. 271 & 2.006 & 0.862 & 4.668 & 0.106 \\
\hline & uc.311 & 0.101 & 0.011 & 0.933 & 0.043 * \\
\hline & uc.376 & 0.412 & 0.191 & 0.888 & 0.024 * \\
\hline & uc. 378 & 0.911 & 0.444 & 1.869 & 0.799 \\
\hline & uc. 427 & 0.569 & 0.247 & 1.312 & 0.186 \\
\hline & uc. 456 & 0.624 & 0.391 & 0.997 & 0.049 * \\
\hline & uc. 475 & 1.035 & 0.616 & 1.738 & 0.897 \\
\hline
\end{tabular}


Table 3. Cont.

\begin{tabular}{|c|c|c|c|c|c|}
\hline \multirow{16}{*}{ 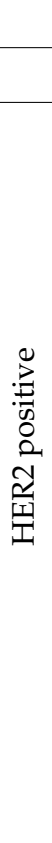 } & \multirow[b]{2}{*}{ Variable } & \multicolumn{4}{|c|}{ Univariate Analysis } \\
\hline & & HR & Lower 0.95 & Upper 0.95 & $p$-Value \\
\hline & Age & 1.073 & 1.030 & 1.119 & $0.001 *$ \\
\hline & Stage & 2.405 & 0.892 & 6.484 & 0.083 \\
\hline & uc. 84 & 2.591 & 0.728 & 9.219 & 0.141 \\
\hline & uc.138 & 1.693 & 0.678 & 4.230 & 0.259 \\
\hline & uc.147 & 6.438 & 0.348 & 119.061 & 0.211 \\
\hline & uc.193 & 2.312 & 1.051 & 5.084 & 0.037 * \\
\hline & uc. 268 & 0.383 & 0.126 & 1.161 & 0.090 \\
\hline & uc. 271 & 1.054 & 0.453 & 2.452 & 0.903 \\
\hline & uc.311 & 1.165 & 0.135 & 10.091 & 0.889 \\
\hline & uc.376 & 1.213 & 0.424 & 3.476 & 0.719 \\
\hline & uc.378 & 0.403 & 0.091 & 1.784 & 0.231 \\
\hline & uc. 427 & 0.478 & 0.132 & 1.736 & 0.262 \\
\hline & uc.456 & 1.266 & 0.600 & 2.674 & 0.535 \\
\hline & uc. 475 & 1.440 & 0.707 & 2.933 & 0.315 \\
\hline
\end{tabular}

${ }^{*} p$-value $<0.05$.

\subsection{T-UCRs Expression in BC Correlates with Patients' Progression Free Interval (PFI)}

In addition to overall survival, we decided to evaluate the patients' progression free interval (PFI) that assesses if the person had a continuation of the cancer, metastasis, or locoregional recurrence and is not polluted with someone who died of other causes than cancer. This analysis was performed with updated TCGA data [30].

Contradictory to overall survival, none of T-UCRs were associated with PFI in general breast cancer when we did the univariate analysis (Table 4). However, when we stratified the TCGA cohort, we found a subtype-specific association between PFI and T-UCRs expression (Table 5).

Table 4. Summary of univariate Cox regression analysis of al T-UCRs in patients' Progression Free Interval.

\begin{tabular}{cccccc}
\hline & \multicolumn{5}{c}{ Univariate Analysis } \\
\cline { 2 - 6 } & Variable & HR & Lower 0.95 & Upper 0.95 & $p$-Value \\
\cline { 2 - 6 } & Age & 1.00 & 0.98 & 1.01 & 0.7399 \\
\cline { 2 - 6 } & Stage & 2.52 & 1.71 & 3.73 & $0.0000^{*}$ \\
\cline { 2 - 6 } & uc.84 & 1.02 & 0.62 & 1.67 & 0.9349 \\
\cline { 2 - 6 } & uc.138 & 1.03 & 0.78 & 1.36 & 0.8447 \\
\cline { 2 - 6 } & uc.147 & 1.00 & 0.28 & 3.52 & 0.9953 \\
\cline { 2 - 6 } & uc.193 & 1.10 & 0.78 & 1.55 & 0.5714 \\
\cline { 2 - 6 } & uc.268 & 1.09 & 0.78 & 1.51 & 0.6127 \\
\cline { 2 - 6 } & uc.271 & 0.91 & 0.67 & 1.23 & 0.5289 \\
\cline { 2 - 6 } & uc.311 & 0.70 & 0.32 & 1.50 & 0.3530 \\
\cline { 2 - 6 } & uc.376 & 1.03 & 0.75 & 1.20 & 0.8574 \\
\cline { 2 - 6 } & uc.378 & 0.87 & 0.63 & 1.34 & 0.7983 \\
\cline { 2 - 6 } & uc.427 & 0.96 & 0.68 & 0.3205 \\
\cline { 2 - 6 } & uc.456 & 1.12 & 0.89 & 0.5769 \\
\hline
\end{tabular}

${ }^{*} p$-value $<0.05$. 
Table 5. Summary of univariate and multivariate Cox regression analysis of T-UCRs in Progression Free Interval among subtypes.

\begin{tabular}{|c|c|c|c|c|c|c|c|c|c|}
\hline & \multirow[b]{2}{*}{ Variable } & \multicolumn{4}{|c|}{ Univariate Analysis } & \multicolumn{4}{|c|}{ Multivariate Analysis } \\
\hline & & HR & Lower 0.95 & Upper 0.95 & $p$-Value & HR & Lower 0.95 & Upper 0.95 & $p$-Value \\
\hline \multirow{14}{*}{ 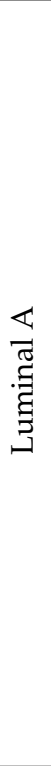 } & Age & 0.996887 & 0.975152 & 1.019106 & 0.78157 & & & & \\
\hline & Stage & 2.105079 & 1.109962 & 3.992354 & $0.019708^{*}$ & 2.76746 & 1.4211 & 5.389 & $0.00276^{*}$ \\
\hline & uc. 84 & 1.979264 & 1.037038 & 3.77757 & $0.037227^{*}$ & 0.0687 & 0.4617 & 2.485 & 0.87288 \\
\hline & uc.138 & 1.542668 & 0.955461 & 2.490761 & 0.075395 & & & & \\
\hline & uc.147 & 4.894229 & 1.028127 & 23.29817 & $0.045626^{*}$ & 0.80186 & 0.3039 & 16.358 & 0.43034 \\
\hline & uc.193 & 1.46312 & 0.737398 & 2.903075 & 0.275879 & & & & \\
\hline & uc. 268 & 1.882928 & 1.069127 & 3.316181 & 0.028443 * & 0.84788 & 1.2133 & 4.493 & 0.01112 * \\
\hline & uc. 271 & 1.022169 & 0.72409 & 1.442955 & 0.900793 & & & & \\
\hline & uc.311 & 2.114192 & 0.699488 & 6.390118 & 0.184278 & & & & \\
\hline & uc.376 & 1.663846 & 0.984975 & 2.810615 & 0.056709 & & & & \\
\hline & uc.378 & 1.062238 & 0.715155 & 1.57777 & 0.764795 & & & & \\
\hline & uc. 427 & 1.508173 & 0.906657 & 2.508762 & 0.113104 & & & & \\
\hline & uc. 456 & 1.839661 & 1.22984 & 2.751864 & $0.003006^{*}$ & 0.62948 & 1.1067 & 3.182 & $0.01947^{*}$ \\
\hline & uc. 475 & 1.522604 & 1.042151 & 2.224555 & 0.029338 * & 0.02543 & 0.5948 & 1.769 & 0.92714 \\
\hline \multirow{15}{*}{ 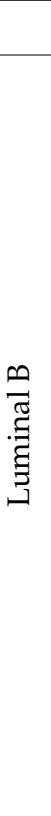 } & & & & & & HR & lower 0.95 & upper 0.95 & $p$-value \\
\hline & Age & 0.999501 & 0.968503 & 1.031491 & 0.97523 & & & & \\
\hline & Stage & 2.052156 & 0.831668 & 5.06373 & 0.111112 & & & & \\
\hline & uc. 84 & 0.156926 & 0.025691 & 0.95853 & 0.04443 * & 0.3566 & 0.060104 & 2.1159 & 0.2564 \\
\hline & uc.138 & 0.735099 & 0.35422 & 1.525523 & 0.408851 & & & & \\
\hline & uc.147 & 0.002767 & $4.9 \times 10^{-5}$ & 0.156281 & $0.002901 *$ & 0.0134 & 0.000186 & 0.9673 & $0.0482 *$ \\
\hline & uc.193 & 1.501734 & 0.65642 & 3.435611 & 0.33617 & & & & \\
\hline & uc. 268 & 0.670959 & 0.31922 & 1.410269 & 0.292864 & & & & \\
\hline & uc. 271 & 1.032127 & 0.318954 & 3.339942 & 0.957908 & & & & \\
\hline & uc.311 & 0.046056 & 0.003497 & 0.606546 & 0.017788 * & 0.2223 & 0.014298 & 3.4558 & 0.2827 \\
\hline & uc.376 & 0.609323 & 0.256861 & 1.445431 & 0.260315 & & & & \\
\hline & uc.378 & 1.3172 & 0.565736 & 2.263933 & 0.72638 & & & & \\
\hline & uc. 427 & 0.540814 & 0.213756 & 1.36829 & 0.191485 & & & & \\
\hline & uc. 456 & 0.671194 & 0.378525 & 1.19015 & 0.171756 & & & & \\
\hline & uc. 475 & 1.136575 & 0.638469 & 2.023284 & 0.663481 & & & & \\
\hline
\end{tabular}


Table 5. Cont.

\begin{tabular}{|c|c|c|c|c|c|c|c|c|c|}
\hline & \multirow[b]{2}{*}{ Variable } & \multicolumn{4}{|c|}{ Univariate Analysis } & \multicolumn{4}{|c|}{ Multivariate Analysis } \\
\hline & & HR & Lower 0.95 & Upper 0.95 & $p$-Value & HR & Lower 0.95 & Upper 0.95 & $p$-Value \\
\hline & & & & & & HR & lower 0.95 & upper 0.95 & $p$-value \\
\hline \multirow{14}{*}{ 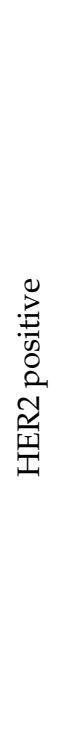 } & Age & 1 & 1 & 1.047071 & 0.910025 & & & & \\
\hline & Stage & 3.246709 & 0.970043 & 10.86666 & $0.044062 *$ & 4.063 & 1.07112 & 15.412 & 0.03931 * \\
\hline & uc. 84 & 1.306121 & 0.257434 & 6.626739 & 0.746998 & & & & \\
\hline & uc.138 & 1.409298 & 0.761768 & 2.607252 & 0.269814 & & & & \\
\hline & uc.147 & 35.01671 & 2.190718 & 559.7114 & $0.008221 *$ & 66.3233 & 3.1042 & 1417.043 & 0.00725 * \\
\hline & uc.193 & 0.738199 & 0.296394 & 1.838563 & 0.514986 & & & & \\
\hline & uc. 268 & 0.576907 & 0.189042 & 1.760575 & 0.333955 & & & & \\
\hline & uc. 271 & 1.067562 & 0.400901 & 2.842817 & 0.895887 & & & & \\
\hline & uc.311 & 1.396482 & 0.10022 & 19.45885 & 0.803706 & & & & \\
\hline & uc.376 & 1.940194 & 0.687189 & 5.477901 & 0.207876 & & & & \\
\hline & uc.378 & 0.072952 & 0.005262 & 1.011354 & $0.047048^{*}$ & 0.1338 & 0.01272 & 1.407 & 0.09385 \\
\hline & uc. 427 & 2.014339 & 0.623733 & 6.505283 & 0.237475 & & & & \\
\hline & uc. 456 & 1.426328 & 0.687533 & 2.959 & 0.33758 & & & & \\
\hline & uc. 475 & 2.404616 & 0.916675 & 6.307773 & 0.070817 & & & & \\
\hline
\end{tabular}

In luminal A, five T-UCRs (uc.84, uc.147, uc.268, uc.456 and uc.475) were associated to PFI in the univariate analysis and four of them (uc.84, uc.147, uc.268, and uc.475) also had an association with PFI in multivariate analysis. The difference here is for the uc.193 that had a greater association with overall survival, but no association with PFI.

According to uni- and multivariate analyses, three T-UCRs (uc.84, uc.147 and uc.311) were correlated to the PFI in luminal B subtype. Hence, uc.84 was associated only with PFI, but not with overall survival. In addition, uc. 147 and uc. 378 were associated with PFI in the HER2 subtype, but only uc.147 continued to have a significant correlation in the multivariate analysis, indicating the high correlation of these T-UCR with the HER2 subtype (Table 5). These results are more specific and provide a more detailed analysis concerning the association of T-UCRs with the survival rate in different subtypes, revealing breast tumor heterogeneity and the relevance of T-UCRs in specific contexts.

\section{Discussion}

Breast cancer is a heterogeneous disease with many clinical and molecular characteristics [15]. Despite the BC general classification system, enhancements to intrinsic subtype classifications need to be proposed to improve prognosis, recurrence risk, survival, or treatment options [31]. Moreover, tumor characteristics can vary according to the population [32].

LncRNAs hold a great promise to be used as biomarkers for diagnosis and prognosis in breast cancer. Studies are already underway to have them developed as the basis of nextgeneration therapeutics for various cancers [33]. Nonetheless, lncRNAs derived from the T-UCRs are underexplored as potential BC biomarkers. In a previous study, we analyzed all 481 T-UCRs in BC TCGA patients, showing that $63 \%$ of transcripts correlated with some clinical and/or molecular parameter of BC.

In the present more in depth analysis, we evaluated $12 \mathrm{~T}$-UCRs according to molecular/clinical parameters in two distinct cohorts: TCGA cohort (827) and Brazilian patients (102).

Uc.84 was associated with PAM50 subtypes with higher expression in luminal A patients. Additionally, it was found to be less expressed in HER-2 positive patients and patients with metastasis events. These results associated uc. 84 with less aggressive tumors. However, this may be a reflection of subtype association. Usually, luminal patients presents 
less metastasis compared to more aggressive subtypes. This idea is supported by the survival analysis only considering luminal A and B patients. In this group, the high expression of uc.84 patients was associated with worse survival time and, in this type of cell, may also be involved in more aggressive pathways. In a recent study, authors showed that a correlation of uc. 84 with mir-221 is associated with the regulation of the CDKND1 and plays pivotal role in the cell cycle in breast cancer cell lines [34].

On the other hand, uc.138 is under-expressed in tumorous tissues compared to nontumorous ones. Uc.138 is less expressed in PR and HER-2 positive samples when associated with subtypes. In luminal B patients, high expression is associated with better survival, but this association is not seen in PFI. Uc.138 was previously associated with tumors, but in colon cancer $[35,36]$. In contrast with our observation in breast cancer, uc.138 is overexpressed and associated with a poor prognosis in colon cancer cases [36]. In colon cells, the transcript containing uc.138 is nuclear and influences cell proliferation by changing the expression of G2/M-related cell cycle regulators.

Uc.147 was previously highlighted by our group as an important lncRNA in breast cancer [26]. The silencing of uc.147 in luminal BC cell lines increases apoptosis, arrests cell cycle, and decreases colony formation and cell viability [26]. These experiments indicated that this T-UCR acts as an oncogene in BC cell lines. Therefore, here, we reinforce the importance of the lnc-uc.147, since this lncRNA was highly expressed in tumor versus non-tumor, in luminal subtypes, including association with ER/PR presence, and had an important association with poor survival in luminal and with PFI in luminal A/B and HER2 patients. The previous study combined with our results increases the potential of uc.147 as a prognostic marker in BC.

Uc.193 is also an important molecule that needs deeper analysis. This T-UCR is associated with tumor in general, molecular subtypes, and ER/PR presence. Uc.193 was associated with poor overall survival considering all BC patients, luminal A, and HER-2 subtypes. However, when we looked at the PFI analysis, the uc.193 did not demonstrate any association with survival, showing a potential confounder with types of death excluding cancer. We found that uc.268 was highly expressed in tumor cells and associated with molecular subtype and ER/PR presence. Additionally, uc.268 is more expressed in diagnosed patients under 40 years age. In luminal A patients, the high expression was associated with a poor survival rate, so apparently the expression of this region is more related to worse features.

Uc.271, uc.378, and uc.427 have a similar expression profile, as they are highly expressed in tumorous tissues compared to non-tumorous ones, associated with molecular subtypes, and are more expressed in PR/ER positive patients. On the other hand, uc.311 and uc.376 were less expressed in tumorous versus non-tumorous tissues, also associated with molecular subtypes and ER/PR positivity.

Uc.456 was hypoexpressed in tumor cells, associated with molecular subtypes, and its high expression was found in ER/PR tumors. Interestingly, in luminal A patients, uc.456 expression was associated with a poor survival rate in OS and PFI analysis. Uc.475 expression was different in molecular subtypes including ER/PR positive patients. In the luminal A group, it was associated with poor prognosis. Uc.475 is considered a hypoxia-induced, noncoding, ultraconserved transcript that is overexpressed in epithelial cancer types [37]. In colon cell lines, under hypoxic conditions, uc. 475 knockdown decreased cell proliferation by G2/M arrest. In breast cancer, the role of this UCR is still under investigated.

An important aspect of the present study is that the diagnostic potential of T-UCRs was evaluated through individual and combined ROC curves. The combination of all 12 studied T-UCRs demonstrated high impact as a diagnostic marker for BC, with an AUC of 0.93 . Another combination of seven T-UCRs (uc.138, uc.147, uc.268, uc.271, uc.311, uc.427, and uc.475) also demonstrated an AUC of 0.92. These results suggest an interesting panel able to differentiate tumorous versus non-tumorous tissue.

Subtype classification is a key determinant for patient outcome and therapeutic choices. In this study, the analysis of T-UCRs levels within two major BC groups, luminal A and 
TNBC, indicated that high expression levels of uc.147 were able to discriminate luminal A patients from TNBC patients with high accuracy (AUC of 0.822 and 0.815 for TCGA and Brazilian cohort, respectively). Interestingly, the discriminatory accuracy of the ROC curve improves when combined with the expression levels of uc.147, uc.271, and uc.427, which also presented good AUC values (0.9531).

This is the first time that the association of T-UCRs with important clinicopathological features was deeply evaluated in BC. More studies are needed to validate the T-UCRs as potential biomarkers in BC as well as other cancers. Nevertheless, this study opens a field to be explored inside the ncRNAs world.

\section{Conclusions}

We presented the T-UCRs uc.84, uc.138, uc.147, uc.193, uc. 268 , uc. 271 , uc.311, uc.376, uc.378, uc.427, uc.456, and uc.475, their association with different clinical and molecular parameters in BC, and their correlation of features in different cohorts. In addition, we demonstrated that T-UCRs could be used to stratify patients inside each subtype in breast cancer and help therapy decisions. Altogether, these results indicate the potential of T-UCRs as a prognostic marker in breast cancer.

Supplementary Materials: The following supporting information can be downloaded at: https: / / www.mdpi.com/article/10.3390/biom12020214/s1, Figure S1: Comparison of T-UCRs expression to estrogen receptor from TCGA data. Figure S2: Comparison of T-UCRs expression to progesterone receptor from TCGA data. Figure S3: Comparison of T-UCRs expression to progesterone receptor from TCGA data. Table S1: Clinical parameters of Brazilian patients. Table S2: Primers sequences used in this study. Table S3: multivariate analysis of Luminal A patients.

Author Contributions: Conceptualization, E.P.Z. and J.C.d.O.; methodology, E.P.Z., C.I, I.R., D.A., D.F.G., E.M.S.F.R. and A.C.R.; software, C.I.; validation, E.P.Z., D.A., G.A.C. and J.C.d.O.; formal analysis, E.P.Z.; investigation, E.P.Z., J.C.d.O. and D.F.G.; resources: E.M.S.F.R., D.F.G., J.C.d.O. and G.A.C.; data curation, J.C.d.O., D.F.G. and E.M.S.F.R.; writing-original draft preparation, E.P.Z.; writing—review and editing, J.C.d.O., D.F.G., E.M.S.F.R., D.A. and G.A.C.; visualization, E.P.Z.; supervision, J.C.d.O. and G.A.C.; project administration, J.C.d.O. and G.A.C.; funding acquisition, J.C.d.O., D.F.G. and E.M.S.F.R. All authors have read and agreed to the published version of the manuscript.

Funding: This research was funded by CNPq/Fundação Araucária: 116/2018, CNPq/Fundação Araucária/Ministério da Saude. PPSUS: 036/2017, and CAPES (001 and CAPES/PRINT grant number 88887.194815/2018-00).

Institutional Review Board Statement: The study was conducted according to the guidelines of the Declaration of Helsinki, and approved by the Ethics Committee of the Federal University of Paraná (CONEP: 19870319.3.0000.0102 8 November 2019).

Informed Consent Statement: Informed consent was obtained from all subjects involved in the study.

Data Availability Statement: The data presented in this study are available on request from the corresponding author.

Acknowledgments: The authors would like to thank the Academic Publishing Advisory Center (Centro de Assessoria de Publicação, CAPA—www.capa.ufpr.br (accessed on 9 January 2022)), of the Federal University of Paraná for assistance with English language translation and editing.

Conflicts of Interest: The authors declare no conflict of interest.

\section{References}

1. Braicu, C.; Zimta, A.A.; Harangus, A.; Iurca, I.; Irimie, A.; Coza, O.; Berindan-Neagoe, I. The Function of Non-Coding RNAs in Lung Cancer Tumorigenesis. Cancers 2019, 11, 605. [CrossRef] [PubMed]

2. Ling, H.; Girnita, L.; Buda, O.; Calin, G.A. Non-coding RNAs: The cancer genome dark matter that matters! Clin. Chem. Lab Med. 2017, 55, 705-714. [CrossRef] [PubMed]

3. Brosnan, C.A.; Voinnet, O. The long and the short of noncoding RNAs. Curr. Opin. Cell Biol. 2009, 21, 416-425. [CrossRef]

4. Bejerano, G.; Pheasant, M.; Makunin, I.; Stephen, S.; Kent, W.J.; Mattick, J.S.; Haussler, D. Ultraconserved elements in the human genome. Science 2004, 304, 1321-1325. [CrossRef] [PubMed] 
5. Mestdagh, P.; Fredlund, E.; Pattyn, F.; Rihani, A.; Van Maerken, T.; Vermeulen, J.; Kumps, C.; Menten, B.; De Preter, K.; Schramm, A.; et al. An integrative genomics screen uncovers ncRNA T-UCR functions in neuroblastoma tumours. Oncogene 2010, 29, 3583-3592. [CrossRef]

6. Calin, G.A.; Liu, C.G.; Ferracin, M.; Hyslop, T.; Spizzo, R.; Sevignani, C.; Fabbri, M.; Cimmino, A.; Lee, E.J.; Wojcik, S.E.; et al. Ultraconserved regions encoding ncRNAs are altered in human leukemias and carcinomas. Cancer Cell 2007, 12, 215-229. [CrossRef] [PubMed]

7. Pereira Zambalde, E.; Mathias, C.; Rodrigues, A.C.; de Souza Fonseca Ribeiro, E.M.; Fiori Gradia, D.; Calin, G.A.; Carvalho de Oliveira, J. Highlighting transcribed ultraconserved regions in human diseases. Wiley Int. Rev. RNA 2020, 11, e1567. [CrossRef]

8. Terracciano, D.; Terreri, S.; de Nigris, F.; Costa, V.; Calin, G.A.; Cimmino, A. The role of a new class of long noncoding RNAs transcribed from ultraconserved regions in cancer. Biochim. Biophys. Acta Rev. Cancer 2017, 1868, 449-455. [CrossRef]

9. Braconi, C.; Valeri, N.; Kogure, T.; Gasparini, P.; Huang, N.; Nuovo, G.J.; Terracciano, L.; Croce, C.M.; Patel, T. Expression and functional role of a transcribed noncoding RNA with an ultraconserved element in hepatocellular carcinoma. Proc. Natl. Acad. Sci. USA 2011, 108, 786-791. [CrossRef]

10. Carotenuto, P.; Fassan, M.; Pandolfo, R.; Lampis, A.; Vicentini, C.; Cascione, L.; Paulus-Hock, V.; Boulter, L.; Guest, R.; Quagliata, L.; et al. Wnt signalling modulates transcribed-ultraconserved regions in hepatobiliary cancers. Gut 2017, 66, 1268-1277. [CrossRef]

11. Luo, H.L.; Chen, J.; Luo, T.; Wu, F.X.; Liu, J.J.; Wang, H.F.; Chen, M.; Li, L.Q.; Li, H. Downregulation of Macrophage-Derived T-UCR uc.306 Associates with Poor Prognosis in Hepatocellular Carcinoma. Cell Physiol. Biochem. 2017, 42, 1526-1539. [CrossRef] [PubMed]

12. Hudson, R.S.; Yi, M.; Volfovsky, N.; Prueitt, R.L.; Esposito, D.; Volinia, S.; Liu, C.G.; Schetter, A.J.; Van Roosbroeck, K.; Stephens, R.M.; et al. Transcription signatures encoded by ultraconserved genomic regions in human prostate cancer. Mol. Cancer 2013, 12, 13. [CrossRef] [PubMed]

13. Olivieri, M.; Ferro, M.; Terreri, S.; Durso, M.; Romanelli, A.; Avitabile, C.; De Cobelli, O.; Messere, A.; Bruzzese, D.; Vannini, I.; et al. Long non-coding RNA containing ultraconserved genomic region 8 promotes bladder cancer tumorigenesis. Oncotarget 2016, 7, 20636-20654. [CrossRef] [PubMed]

14. Bray, F.; Ferlay, J.; Soerjomataram, I.; Siegel, R.L.; Torre, L.A.; Jemal, A. Global cancer statistics 2018: GLOBOCAN estimates of incidence and mortality worldwide for 36 cancers in 185 countries. CA Cancer J. Clin. 2018, 68, 394-424. [CrossRef]

15. Harbeck, N.; Penault-Llorca, F.; Cortes, J.; Gnant, M.; Houssami, N.; Poortmans, P.; Ruddy, K.; Tsang, J.; Cardoso, F. Breast cancer Nat. Rev. Dis. Primers 2019, 5, 66. [CrossRef]

16. Goldhirsch, A.; Wood, W.C.; Coates, A.S.; Gelber, R.D.; Thürlimann, B.; Senn, H.J.; Members, P. Strategies for subtypes-Dealing with the diversity of breast cancer: Highlights of the St. Gallen International Expert Consensus on the Primary Therapy of Early Breast Cancer 2011. Ann. Oncol. 2011, 22, 1736-1747. [CrossRef]

17. Goldhirsch, A. Personalized adjuvant therapies: Lessons from the past: The opening address by the St. Gallen 2013 award recipient. Breast 2013, 22 (Suppl. 2), S3-S7. [CrossRef]

18. Beniey, M.; Haque, T.; Hassan, S. Translating the role of PARP inhibitors in triple-negative breast cancer. Oncoscience 2019, 6, 287-288. [CrossRef]

19. Network, C.G.A. Comprehensive molecular portraits of human breast tumours. Nature 2012, 490, 61-70. [CrossRef]

20. Perou, C.M.; Sørlie, T.; Eisen, M.B.; van de Rijn, M.; Jeffrey, S.S.; Rees, C.A.; Pollack, J.R.; Ross, D.T.; Johnsen, H.; Akslen, L.A.; et al. Molecular portraits of human breast tumours. Nature 2000, 406, 747-752. [CrossRef]

21. Sørlie, T.; Perou, C.M.; Tibshirani, R.; Aas, T.; Geisler, S.; Johnsen, H.; Hastie, T.; Eisen, M.B.; van de Rijn, M.; Jeffrey, S.S.; et al Gene expression patterns of breast carcinomas distinguish tumor subclasses with clinical implications. Proc. Natl. Acad. Sci. USA 2001, 98, 10869-10874. [CrossRef] [PubMed]

22. Andre, F.; Ismaila, N.; Stearns, V. Use of Biomarkers to Guide Decisions on Adjuvant Systemic Therapy for Women With Early-Stage Invasive Breast Cancer: ASCO Clinical Practice Guideline Update Summary. J. Oncol. Pract. 2019, 15, $495-497$. [CrossRef]

23. Curigliano, G.; Burstein, H.J.; Winer, E.P.; Gnant, M.; Dubsky, P.; Loibl, S.; Colleoni, M.; Regan, M.M.; Piccart-Gebhart, M.; Senn, H.J.; et al. De-escalating and escalating treatments for early-stage breast cancer: The St. Gallen International Expert Consensus Conference on the Primary Therapy of Early Breast Cancer 2017. Ann. Oncol. 2017, 28, 1700-1712. [CrossRef] [PubMed]

24. Zhang, L.X.; Xu, L.; Zhang, C.H.; Lu, Y.H.; Ji, T.H.; Ling, L.J. uc.38 induces breast cancer cell apoptosis via PBX1. Am. J. Cancer Res. 2017, 7, 2438-2451.

25. Marini, A.; Lena, A.M.; Panatta, E.; Ivan, C.; Han, L.; Liang, H.; Annicchiarico-Petruzzelli, M.; Di Daniele, N.; Calin, G.A.; Candi, E.; et al. Ultraconserved long non-coding RNA uc.63 in breast cancer. Oncotarget 2016, 8, 35669-35680. [CrossRef]

26. Pereira Zambalde, E.; Bayraktar, R.; Schultz Jucoski, T.; Ivan, C.; Rodrigues, A.C.; Mathias, C.; Knutsen, E.; Silveira de Lima, R.; Fiori Gradia, D.; de Souza Fonseca Ribeiro, E.M.; et al. A novel lncRNA derived from an ultraconserved region: Lnc-uc.147, a potential biomarker in luminal A breast cancer. RNA Biol. 2021, 13, 1-14. [CrossRef]

27. Shi, X.; Huang, X.; Chen, R.; Li, Y.; Xu, Y.; Zhang, W.; Zhu, Q.; Zha, X.; Wang, J. The transcribed ultraconserved element uc.51 promotes the proliferation and metastasis of breast cancer by stabilizing NONO. Clin. Exp. Metastasis 2021, 38, 551-571. [CrossRef] 
28. Li, J.; Han, L.; Roebuck, P.; Diao, L.; Liu, L.; Yuan, Y.; Weinstein, J.N.; Liang, H. TANRIC: An Interactive Open Platform to Explore the Function of lncRNAs in Cancer. Cancer Res. 2015, 75, 3728-3737. [CrossRef]

29. Ciriello, G.; Gatza, M.L.; Beck, A.H.; Wilkerson, M.D.; Rhie, S.K.; Pastore, A.; Zhang, H.; McLellan, M.; Yau, C.; Kandoth, C.; et al. Comprehensive Molecular Portraits of Invasive Lobular Breast Cancer. Cell 2015, 163, 506-519. [CrossRef]

30. Liu, J.; Lichtenberg, T.; Hoadley, K.A.; Poisson, L.M.; Lazar, A.J.; Cherniack, A.D.; Kovatich, A.J.; Benz, C.C.; Levine, D.A.; Lee, A.V.; et al. An Integrated TCGA Pan-Cancer Clinical Data Resource to Drive High-Quality Survival Outcome Analytics. Cell 2018, 173, 400-416. [CrossRef]

31. Burzio, V.A.; Villota, C.; Villegas, J.; Landerer, E.; Boccardo, E.; Villa, L.L.; Martínez, R.; Lopez, C.; Gaete, F.; Toro, V.; et al. Expression of a family of noncoding mitochondrial RNAs distinguishes normal from cancer cells. Proc. Natl. Acad. Sci. USA 2009, 106, 9430-9434. [CrossRef] [PubMed]

32. Hamam, R.; Hamam, D.; Alsaleh, K.A.; Kassem, M.; Zaher, W.; Alfayez, M.; Aldahmash, A.; Alajez, N.M. Circulating microRNAs in breast cancer: Novel diagnostic and prognostic biomarkers. Cell Death Dis. 2017, 8, e3045. [CrossRef] [PubMed]

33. Tomar, D.; Yadav, A.S.; Kumar, D.; Bhadauriya, G.; Kundu, G.C. Non-coding RNAs as potential therapeutic targets in breast cancer. Biochim. Biophys. Acta Gene Regul. Mech. 2020, 1863, 194378. [CrossRef] [PubMed]

34. Corrà, F.; Crudele, F.; Baldassari, F.; Bianchi, N.; Galasso, M.; Minotti, L.; Agnoletto, C.; Di Leva, G.; Brugnoli, F.; Reali, E.; et al. UC.183, UC.110, and UC.84 Ultra-Conserved RNAs Are Mutually Exclusive with miR-221 and Are Engaged in the Cell Cycle Circuitry in Breast Cancer Cell Lines. Genes 2021, 12, 1978. [CrossRef]

35. Kajita, K.; Kuwano, Y.; Satake, Y.; Kano, S.; Kurokawa, K.; Akaike, Y.; Masuda, K.; Nishida, K.; Rokutan, K. Ultraconserved region-containing Transformer $2 \beta 4$ controls senescence of colon cancer cells. Oncogenesis 2016, 5, e213. [CrossRef]

36. Kuwano, Y.; Nishida, K.; Rokutan, K. Overexpression of the transcribed ultraconserved region Uc.138 accelerates colon cancer progression. Sci. Rep. 2021, 11, 8667. [CrossRef]

37. Ferdin, J.; Nishida, N.; Wu, X.; Nicoloso, M.S.; Shah, M.Y.; Devlin, C.; Ling, H.; Shimizu, M.; Kumar, K.; Cortez, M.A.; et al. HINCUTs in cancer: Hypoxia-induced noncoding ultraconserved transcripts. Cell Death Differ. 2013, 20, 1675-1687. [CrossRef] 